\title{
ALGEBRAIC INTEGRABILITY OF THE TWO-BODY RUIJSENAARS OPERATOR
}

\author{
GIOVANNI FELDER ${ }^{*, 1}$ AND ALEXANDER VARCHENKO ${ }^{* *, 2}$ \\ * Centre Emile Borel, Institut Henri Poincaré, Université Pierre et Marie Curie, \\ 75231 Paris Cedex 05, France \\ ** Department of Mathematics, University of North Carolina at Chapel Hill, \\ Chapel Hill, NC 27599-3250, USA
}

October 1996

\begin{abstract}
We study the algebra of difference operators that commute with the two-body Ruijsenaars operator, a $q$-deformation of the Lamé differential operator, for generic values of the deformation parameter. The algebra is commutative. It is the algebra of polynomial functions on an affine hyperelliptic curve $Y^{2}=P\left(X^{2}\right)$. We also compute the difference Galois group of the eigenvalue problem.
\end{abstract}

\section{INTRODUCTION}

We study the eigenvalue problem $L \psi=\epsilon \psi$ for the " $q$-Lamé" difference operator $L$ in one complex variable $\lambda$ :

$$
L \psi(\lambda)=\frac{\theta(\lambda-\gamma m, \tau)}{\theta(\lambda, \tau)} \psi(\lambda+\gamma)+\frac{\theta(\lambda+\gamma m, \tau)}{\theta(\lambda, \tau)} \psi(\lambda-\gamma) .
$$

Here $\gamma$ is a complex parameter, $\tau$ is a parameter in the upper half plane, and $m$ is a nonnegative integer.

The theta function is Jacobi's $\theta_{1}$ function

$$
\theta(z, \tau)=-\sum_{j \in \mathbb{Z}+\frac{1}{2}} e^{\pi i j^{2} \tau+2 \pi i j\left(z+\frac{1}{2}\right)} .
$$

The difference operator $L$ appears in Sklyanin's work [S] on the representation theory of his elliptic algebra. In fact, if we replace the theta functions in the numerators by the other three Jacobi functions $\theta_{\alpha}$, we get difference operators that obey with $L$ the commutation relations of Sklyanin's algebra.

\footnotetext{
${ }^{1}$ Permanent address: D-MATH, ETH-Zentrum, 8092 Zürich, Switzerland.

${ }^{2}$ Supported in part by NSF grant DMS-9501290.
} 
The operator $L$ also appears as the simplest non trivial quantum relativistic Calogero-Moser Hamiltonian, introduced by Ruijsenaars $\mathbb{R}]$ : $L$ is conjugated to the Ruijsenaars two-body Hamiltonian, and its semiclassical (or nonrelativistic) version is the Lamé differential operator. More precisely, as $\gamma \rightarrow 0, L=2 \mathrm{Id}+$ const $\gamma^{2} \ell+O\left(\gamma^{4}\right)$, and $\ell$ is conjugated to

$$
-\frac{d^{2}}{d \lambda^{2}}+m(m+1) \wp(\lambda)+\text { const }
$$

the Lamé differential operator of the classical theory of ellipsoidal harmonics, see [WW]. Here $\wp$ denotes Weierstrass' doubly periodic function with periods 1 and $\tau$.

The eigenvalue problem for the Lamé operator has been studied in the classical literature. In particular, Hermite gave a formula for eigenfunctions which we would call today of "Bethe ansatz" type [WW]: he wrote a simple function depending on $m$ parameters, and proved that this function is an eigenfunction if the parameters are solutions of a set of $m-1$ algebraic equations (or $m$ equations if one wants to specify the eigenvalue). He also showed that all eigenfunctions with generic eigenvalue are linear combinations of eigenfunctions obtained by this construction.

In more modern terminology, the Lamé operator is an example of an "algebraically integrable" or "finite gap" Schrödinger operator. The meaning of this is the content of the following theorem, which follows from the classical results, but whose true paternity appears difficult to establish, see DMN, CV.

Theorem 1.1. There exists a differential operator $\ell_{2 m+1}$ of order $2 m+$ 1, such that the algebra $A$ of differential operators with meromorphic coefficients commuting with $\ell$ is generated by $\ell$ and $\ell_{2 m+1}$. Moreover $A$ is commutative and $\ell \mapsto x, \ell_{2 m+1} \mapsto y$ defines an isomorphism from $A$ to $\mathbb{C}[x, y] /\left(y^{2}-p(x)\right) \mathbb{C}[x, y]$, for some polynomial $p$ of degree $2 m+1$.

In other words the algebra of differential operators commuting with the Lamé operator is isomorphic to the algebra of polynomial functions on an affine hyperelliptic curve. This curve is called the spectral curve of the differential operator $\ell$. Hermite's results can be rephrased as saying that the spectral curve is birational to a subvariety of the $m^{\text {th }}$ symmetric power of the elliptic curve $E_{\tau}=\mathbb{C} / \mathbb{Z}+\tau \mathbb{Z}$.

In [KZ, Krichever and Zabrodin considered the operator $L$, and showed that eigenfunctions are parametrized by points on a hyperelliptic curve.

In [FV], we showed that $L$ is proportional to the transfer matrix of the $2 m+1$ irreducible representation of the elliptic quantum group 
ALGEBRAIC INTEGRABILITY OF THE TWO-BODY RUIJSENAARS OPERATOR

$E_{\tau, \gamma / 2}\left(s l_{2}\right)$. The Bethe ansatz for this transfer matrix generalizes Hermite's results to the difference case, see [FV], Theorem 3.1, and gives a formula for eigenfunctions of $L$ parametrized by a Hermite-Bethe curve $Y$ given by $m-1$ equations for $m$ variables on the elliptic curve $E_{\tau}$. In the formulation of [KZ], we characterize double Bloch functions in terms of their zeros. The results in [KZ] and [FV] are complementary: an "additive" formula for eigenfunctions is given in [KZ], while a "multiplicative" formula is given in [FV]. In both cases the formula depend on parameters on a hyperelliptic curve.

In this paper, we study the difference operators commuting with $L$. Our result is the following. Let $D_{\gamma}$ be the algebra of difference operators of the form

$$
M \psi(\lambda)=\sum_{j=a}^{b} B_{j}(\lambda) \psi(\lambda+j \gamma), \quad a \leq b \in \mathbb{Z}
$$

with 1-periodic meromorphic coefficients $B_{j}$. If $B_{a}$ and $B_{b}$ are non zero, we say that $M$ has degree $b$ and length $b-a$. The degree and the length of a product is the sum of the degrees and of the lengths of the factors, respectively.

Theorem 1.2. Suppose that $\gamma$ is an irrational number. Then there exists a difference operator $N$ of degree $2 m+1$ and length $4 m+2$, such that the algebra $A$ of operators in $D_{\gamma}$ commuting with $L$ is generated by $L$ and $N$. Moreover $A$ is commutative and $L \mapsto X, N \mapsto Y$ defines an isomorphism from $A$ to $\mathbb{C}[X, Y] /\left(Y^{2}-P\left(X^{2}\right)\right) \mathbb{C}[X, Y]$, for some polynomial $P$ of degree $2 m+1$.

In the difference case, the spectral curve has two involutive automorphisms. The automorphism $(X, Y) \mapsto(X,-Y)$ corresponds to the symmetry $S \psi(\lambda)=\psi(-\lambda)$ of the eigenvalue problem, as in the differential case. The automorphism $(X, Y) \mapsto(-X,-Y)$ corresponds to the involution $U S \psi(\lambda)=e^{\pi i \lambda / \gamma} \psi(-\lambda)$ which maps eigenfunctions to eigenfunctions with opposite eigenvalue.

The operator $N$ can be written explicitly in terms of a remarkable family of difference operators $M_{l}$ commuting with $L$ :

Theorem 1.3. Suppose that $\gamma \neq 0 \bmod \mathbb{Z}+\tau \mathbb{Z}$. For each generic $l \in \mathbb{C}$ there exists a difference operator $M_{l}$ of the form

$$
M_{l} \psi(\lambda)=\sum_{k=0}^{m} A_{l-m+2 k}^{l}(\lambda / \gamma) \psi(\lambda+(l-m+2 k) \gamma)
$$


These operators commute pairwise and obey the relations

$$
L M_{l}=\frac{\theta(\gamma(l-m), \tau)}{\theta(\gamma l, \tau)} M_{l+1}+\frac{\theta(\gamma(l+m), \tau)}{\theta(\gamma l, \tau)} M_{l-1},
$$

and the product rules

$$
M_{l} M_{k}=\sum_{j} A_{j}^{l}(k) M_{k+j} .
$$

Moreover, $L$ is proportional to $M_{m-1}$ and $N$ is proportional to $M_{m+1}-$ $M_{-m-1}$.

We can then say, up to change of variable, that $M_{l}$ is an operator eigenfunction of the Lamé operator with "eigenvalue" $L$.

The eigenvalue problem $L \psi=\epsilon \psi$ becomes, after the change of independent variable $\psi(\lambda)=u(\lambda) \prod_{j=1}^{m} \theta(\lambda-j \gamma)$,

$$
u(\lambda+\gamma)+\frac{\theta(\lambda+\gamma m, \tau) \theta(\lambda-\gamma(m+1), \tau)}{\theta(\lambda, \tau) \theta(\lambda-\gamma, \tau)} u(\lambda-\gamma)=\epsilon u(\lambda) .
$$

This equation has elliptic coefficients. Note incidentally that if $\gamma \in$ $\mathbb{Z}+\tau \mathbb{Z}$, the equation has constant coefficients and can be easily solved in terms of exponential functions.

In the last section of this paper we compute the difference Galois group, an analogue of the differential Galois group of differential equations, of this equation in the case of irrational $\gamma$. It is the Galois group of the extension of the difference field of elliptic functions generated by the solutions. It turns out that this Galois group is Abelian, in agreement with the philosophy of [BEG], who characterized algebraically integrable differential operators by the Abelian nature of the corresponding differential Galois groups.

\section{Elliptic NUMBer NOTATION}

We assume that $\gamma \neq 0 \bmod \mathbb{Z}+\tau \mathbb{Z}$. It is convenient to introduce a new variable $x=\lambda / \gamma$. In this variable, the step of the difference operator $L$ is integer, and the periods are $\omega=\gamma^{-1}$ and $\omega^{\prime}=\tau \gamma^{-1}$. The "elliptic number"

$$
[x]=\frac{\theta(\gamma x, \tau)}{\theta(\gamma, \tau)}
$$

is an odd entire function of $x$ with zeros on the lattice $\mathbb{Z} \omega+\mathbb{Z} \omega^{\prime}$ and has transformation properties

$$
[x+\omega]=-[x], \quad\left[x+\omega^{\prime}\right]=-e^{-\pi i \tau-2 \pi i \gamma x}[x]=-e^{-\frac{\pi i}{\omega}\left(\omega^{\prime}+2 x\right)}[x] .
$$


ALGEBRAIC INTEGRABILITY OF THE TWO-BODY RUIJSENAARS OPERATOR

In this variable, which we use in the rest of this paper, the $q$-Lamé operator reads

$$
L=\frac{[x-m]}{[x]} T_{1}+\frac{[x+m]}{[x]} T_{-1}, \quad T_{j} \psi(x)=\psi(x+j) .
$$

It will also be convenient to introduce "elliptic binomial coefficients" and "elliptic factorials":

$$
\left[\begin{array}{l}
x \\
n
\end{array}\right]=\frac{[x][x-1] \cdots[x-n+1]}{[n][n-1] \cdots[1]}, \quad\left[\begin{array}{l}
x \\
0
\end{array}\right]=1, \quad[n] !=[1][2] \cdots[n] .
$$

\section{Bethe EIGENFUnCTIONS}

We review the results of $[\mathrm{FV}$ on the $q$-Lamé operator. We assume that $\gamma \neq 0 \bmod \mathbb{Z}+\tau \mathbb{Z}$. Let us introduce $m$ functions of $t=\left(t_{1}, \ldots, t_{m}\right) \in \mathbb{C}^{m}:$

$$
b_{i}(t)=\frac{\left[t_{i}-m\right]}{\left[t_{i}+m\right]} \prod_{j: j \neq i} \frac{\left[t_{j}-t_{i}-1\right]}{\left[t_{j}-t_{i}+1\right]}, \quad i=1, \ldots, m .
$$

Theorem 3.1. [FV Let $\left(t_{1}, \ldots, t_{m}, c\right)$ be a solution of the Bethe ansatz equations:

$$
b_{i}(t)=e^{2 \gamma c}, \quad i=1, \ldots, m,
$$

such that $t_{i} \neq t_{j} \bmod \omega \mathbb{Z}+\omega^{\prime} \mathbb{Z}$, if $i \neq j$. Then

$$
\psi(x)=e^{c \gamma x} \prod_{j=1}^{m}\left[x+t_{j}\right]
$$

is a solution of the $q$-Lamé equation $\frac{[x-m]}{[x]} \psi(x+1)+\frac{[x+m]}{[x]} \psi(x-1)=$ $\epsilon_{L} \psi(x)$, with eigenvalue

$$
\epsilon_{L}(t)=e^{-\gamma c} \frac{[2 m]}{[m]} \prod_{j=1}^{m} \frac{\left[t_{j}+m-1\right]}{\left[t_{j}+m\right]} .
$$

Remark. What was called $t_{j}$ in [FV] is here $\left(t_{j}-1 / 2\right) \gamma$. Also, $\gamma=2 \eta$ in the notation of [FV].

Note that the functions $b_{j}$ have the propery that, for all $i, j$,

$$
\begin{aligned}
b_{j}\left(t_{1}, \ldots, t_{i}+\omega, \ldots, t_{m}\right) & =b_{j}\left(t_{1}, \ldots, t_{i}, \ldots, t_{m}\right), \\
b_{j}\left(t_{1}, \ldots, t_{i}+\omega^{\prime}, \ldots, t_{m}\right) & =e^{4 \pi i \gamma} b_{j}\left(t_{1}, \ldots, t_{i}, \ldots, t_{m}\right) .
\end{aligned}
$$

Thus if $\left(t_{1}, \ldots, t_{m}, c\right)$ is a solution then $\left(t_{1}, \ldots, t_{i}+\omega, \ldots, c\right)$ and $\left(t_{1}, \ldots, t_{i}+\right.$ $\left.\omega^{\prime}, \ldots, c+2 \pi i\right)$ are also solutions. Moreover, the eigenfunctions are the same for these solutions. Also, the equations and the eigenfunctions are symmetric under permutations of the $t_{j}^{\prime} s$. We thus have an action 
of the semidirect product $\Gamma=\left(\omega \mathbb{Z}+\omega^{\prime} \mathbb{Z}\right)^{m} \tilde{\times} S_{m}$ on $\mathbb{C}^{m} \times \mathbb{C}$, and the Bethe ansatz equations take place in the quotient: The set of solutions is

$$
\begin{aligned}
X_{\infty} & =\left\{(t, c) \in\left(\mathbb{C}^{m}-D\right) \times \mathbb{C} \mid b_{j}(t)=e^{2 \gamma c}, j=1, \ldots, m\right\} / \Gamma, \\
D & =\cup_{i<j}\left\{t_{i}=t_{j} \quad \bmod \omega \mathbb{Z}+\omega^{\prime} \mathbb{Z}\right\} .
\end{aligned}
$$

On $X_{\infty}$ we have a $\mathbb{Z}$-action $(t, c) \mapsto(t, c+\pi i / \gamma)$. The quotient is the set

$$
X=X_{\infty} / \mathbb{Z}
$$

determined by the equations $b_{i}(t)=b_{j}(t)$. It is an algebraic subvariety of the symmetric power of our elliptic curve. The eigenvalue $\epsilon_{L}$ is a two-valued function on $X$. It is a single-valued meromorphic function on the two-fold covering

$$
X_{2}=X_{\infty} / 2 \mathbb{Z}
$$

The eigenfunctions associated to points in $X_{\infty}$ which have the same projection on $X_{2}$ are proportional, in the sense that they are related by multiplication by a 1-periodic function.

In other words, a point in $X_{2}$ parametrizes a one-dimensional vector space of eigenfunctions over the field $K$ of 1-periodic meromorphic functions. Note that these eigenfunctions have all the same eigenvalue.

Eigenfunctions associated to points in $X_{2}$ with the same projection on $X$ are related by the map $U: \psi \mapsto e^{\pi i x} \psi$. They have opposite eigenvalues.

These varieties can be described rather explicitly in the neighborhood of $c=\infty$ :

Lemma 3.2. [FV Let $\bar{X}$ be the closure of $X$ in the symmetric power $S^{m} E$ of the elliptic curve $\mathbb{C} / \omega \mathbb{Z}+\omega^{\prime} \mathbb{Z}$. Then $\bar{X}$ contains the two points $P_{+}=(-m+1, \ldots,-1,0)$ and $P_{-}=(m-1, \ldots, 1,0)$. The irreducible component(s) of $X$ containing $P_{+}$and $P_{-}$form a curve $Y$ which is smooth at these two points. In terms of a local coordinate vanishing at $P_{+}$, the eigenvalue has the form $\epsilon_{L}=$ const $u^{-1 / 2}(1+O(u))$, and $e^{2 c \gamma}=$ const $u^{-1}+O(1)$.

Let $Y_{2} \subset X_{2}$ be the double covering of $Y$. It is a curve on which the eigenvalue is a single-valued function. The two points $P_{+}, P_{-}$ are related by the symmetry $(t, c) \mapsto(-t,-c)$ of the Bethe ansatz equations.

Theorem 3.3. [FV Suppose that $\gamma \in \mathbb{C}$ is generic. For generic $\in \in \mathbb{C}$, there are precisely two solutions

$$
\left(t_{1}, \ldots, t_{m}, c\right) \quad \text { and } \quad\left(-t_{1}, \ldots,-t_{m},-c\right)
$$


ALGEBRAIC INTEGRABILITY OF THE TWO-BODY RUIJSENAARS OPERATOR of the Bethe ansatz equations (1) in $X_{\infty}$ with given eigenvalue $\epsilon_{L}=\epsilon$. The corresponding eigenfunctions $\psi_{ \pm}$are linearly independent over the field $K$ of 1-periodic meromorphic functions of $x$, and all solutions of the $q$-Lamé equation $L \psi=\epsilon_{L} \psi$ are linear combinations of $\psi_{+}, \psi_{-}$with coefficients in $K$.

Definition: The (many-valued) function on $X \times \mathbb{C}$

$$
\psi(t, x)=e^{c \gamma x} \prod_{j=1}^{m} \frac{\left[x+t_{j}\right]}{\left[t_{j}\right]}
$$

where $c$ is determined by the Bethe ansatz equations, is called the Baker-Akhiezer function.

The Baker-Akhiezer function is single-valued on $X_{\infty} \times \mathbb{C}$.

Lemma 3.4. If $M=\sum_{j} A_{j}(x) T_{j}$ is a difference operator with finitely many non-zero coefficients $A_{j}$ such that $M \psi(t, \cdot)=0$ for all $t$ on the curve $Y$, then $M=0$.

Proof: We consider the equation $M \psi(t, x)=0$ in the vicinity of the point $P_{+}$of Lemma 3.2: let $k$ be the largest number so that $A_{k} \neq 0$. Since $\psi(t, x+j) / \psi(t, x)$ behaves as $e^{\gamma c j} \sim u^{-j / 2}$ as $u \rightarrow 0$, we see that for $M \psi / \psi$ to vanish, it is necessary that its leading coefficient $A_{k}(x)$ vanishes, a contradiction. Thus $M=0$.

\section{Difference operators COMmuting With the $q$-Lamé OPERATOR}

We construct a sequence of difference operators that commute with the $q$-Lamé operator $L$. Let

$$
M_{l}=A_{l-m}^{l}(x) T_{l-m}+A_{l-m+2}^{l}(x) T_{l-m+2}+\cdots+A_{l+m}^{l}(x) T_{l+m}
$$

Usually $l$ is an integer, but we will occasionally take $l$ to be a general complex number. For $k=0, \ldots, m$,

$$
\begin{aligned}
A_{l-m+2 k}^{l}(x)= & (-1)^{k}\left[\begin{array}{c}
m \\
k
\end{array}\right] \prod_{j=0}^{m-k-1} \frac{[l+m-j][x+m-j]}{[x+l+k-j]} \\
& \times \prod_{j=0}^{k-1} \frac{[l-m+j][x-m+j]}{[x+l-m+k+j]},
\end{aligned}
$$

with the understanding that a product over the empty set is one.

\section{Theorem 4.1.}

(i) $M_{m}=\frac{[2 m] !}{[m] !} \mathrm{Id}, M_{m-1}=\frac{[2 m-1] !}{[m-1] !} L$. 
(ii) For all complex numbers $l, k, M_{l} M_{k}=M_{k} M_{l}$.

Proof: (i) If $l=m$, all coefficient $A_{l-m+2 k}^{l}$ with $k \neq 0$ vanish because of the factor $[l-m]$ in the second product. If $k=0$,

$$
A_{0}^{m}(x)=\left[\begin{array}{c}
m \\
0
\end{array}\right] \prod_{j=0}^{m-1}[2 m-j]=\frac{[2 m] !}{[m] !}
$$

Similarly, if $l=m-1$, the only non-vanishing coefficients are $A_{1}^{l}(x)=$ $[2 m-1] ![x-m] /[m-1] ![x]=A_{-1}^{l}(-x)$.

(ii) We first show that $M_{l}$ commutes with the $q$-Lamé operator $L$. The equation $M L=L M$ for an operator of the form $M=\sum A_{j} T_{j}$ is equivalent to the identities

$A_{j}(x) \frac{[x+j+m]}{[x+j]}+A_{j-2}(x) \frac{[x+j-2-m]}{[x+j-2]}=A_{j}(x-1) \frac{[x+m]}{[x]}+A_{j-2}(x+1) \frac{[x-m]}{[x]}$,

for its coefficients. We have to show that our $A_{j}^{l}$, which are zero except for $j$ in the set $\{l-m, l-m+2, \ldots, l+m\}$, are a solution of this equation. If we insert the formulae, and cancel common factors, we see that the identity we have to prove is

$$
\begin{gathered}
\frac{[l-m+k-1][x-m+k-1][x+l+2 k]}{[k][x+l+k]}-\frac{[l+k][x+k][x+l+2 k-2 m-2]}{[m-k+1][x+l+k-m-1]} \\
=\frac{[l-m+k-1][x+k][x-m-1][x+l+2 k-m-1]}{[k][x][x+l+k-m-1]} \\
-\frac{[l+k][x+m+1][x+k-m-1][x+l+2 k-m-1]}{[m-k+1][x][x+l+k]} .
\end{gathered}
$$

We make use of the properties of elliptic numbers under translations by $\omega=\gamma^{-1}$ and $\omega^{\prime}=\gamma^{-1} \tau$. Dividing both sides of this equation by $[x+k]$ yields an equation, such that all terms are periodic functions of $x$ with period $\omega$ and are multiplied by $\exp (2 \pi i \gamma(m-k+1))$, if $x$ is replaced by $x+\omega^{\prime}$. The (simple) poles are at $x=0,-l-k,-k$ and $-l+m-k+1$. However, the difference between the left-hand side and right-hand side has vanishing residue at these poles, as is easily checked, for any generic value of $k$. But if $\alpha$ is a generic complex number, the only entire holomorphic function $f$ such that $f(z+\omega)=f(z)$ and $f\left(z+\omega^{\prime}\right)=\alpha f(z)$ is $f=0$. Thus the identity is proved for generic, and, by analiticity for all, values of $k$.

To complete the proof of the commutativity of $M_{l}$ and $L$ we have to check the identity for the coefficients separately in the two extreme cases $j=l-m$ and $j=l+m+2$, for which only two terms are non-zero. This is easily done. 
ALGEBRAIC INTEGRABILITY OF THE TWO-BODY RUIJSENAARS OPERATOR

This completes the proof of (ii) if $l=m-1$. To prove the general case, note that for any point $t \in X$,

$$
L M_{l} \psi(t, \cdot)=M_{l} L \psi(t, \cdot)=\epsilon_{L}(t) M_{l} \psi,(t, \cdot) .
$$

Hence $M_{l} \psi(t, \cdot)$ is an eigenfunction of $L$ with eigenvalue $\epsilon_{L}(t)$. By Theorem 3.3, it is proportional to $\psi(t, \cdot)$. The same holds for $M_{k}$. It follows that for generic $t,\left[M_{l}, M_{k}\right] \psi(t, \cdot)=0$. Lemma 3.4 implies then that $\left[M_{l}, M_{k}\right]=0$.

Lemma 4.2. Let $S$ and $U$ be the operators $S \psi(x)=\psi(-x)$ and $U \psi(x)=$ $e^{\pi i x} \psi(x)$. Then, for all $l, M_{l} S=S M_{-l}$ and $U M_{l}=e^{\pi i(l-m)} M_{l} U$.

Proof: The first statement follows from the relation $T_{j} S=S T_{-j}$ and the identity

$$
A_{j}^{l}(x)=A_{-j}^{-l}(-x)
$$

The second follows from the relation $U T_{l-m+2 k}=e^{\pi i(l-m)} T_{l-m+2 k} U$.

Lemma 4.3. Suppose that $\gamma$ is irrational and let $\omega=1 / \gamma$. Let $\phi(x)=$ $\prod_{k=1}^{m}[x-k]$. Then, for any $j \in \mathbb{C}$, the first coefficient of any difference operator $M=\sum_{k=0}^{l} B_{j-k}(x) T_{j-k}$ with $\omega$-periodic coefficients $B_{j-k}$ which commutes with $L$ has the form $B_{j}(x)=c \frac{\phi(x)}{\phi(x+j)}$ for some constant c.

Proof: The $q$-Lamé operator $L$ can be written in the form

$$
L=\phi(x) T_{1} \phi(x)^{-1}+\phi(-x) T_{-1} \phi(-x)^{-1} .
$$

It follows that $M_{\phi}=\phi^{-1} M \phi$ commutes with $T_{1}+C(x) T_{-1}$ for some $C$. Thus the coefficient of $T_{j}$ in $M_{\phi}$ obeys the difference equation $T_{1} f(x)=f(x)$ or $f(x+1)=f(x)$. But $f$ is also $\gamma^{-1}$-periodic since $M$ is in $D_{\gamma}$. If $\gamma$ is irrational, it follows that $f$ is a constant $c$. Thus $M=c \phi(x) T_{j} \phi(x)^{-1}+\cdots=c \phi(x) \phi(x+j)^{-1} T_{j}+\cdots$.

Lemma 4.4. Let $\gamma$ be irrational. If $l \geq m+1$ or if $l \leq-m-1$ then the $M_{l}$ has degree $l+m$ and length $2 m$. If $l \in\{-m,-m+1, \ldots, m\}$ then $M_{l}$ has degree $|m-l|$ and length $2|m-l|$.

Proof: This amounts to check when the coefficients $A_{k}^{l}$ vanish, which is easy, given their factorized form.

Lemma 4.5. Suppose that $\gamma$ is irrational and let $\omega=1 / \gamma$. Suppose that $j$ is either a generic complex number or a negative integer. Then, any difference operator commuting with $L$ of the form

$$
M=B_{j}(x) T_{j}+B_{j-2}(x) T_{j-2}+\cdots+B_{j-2 l}(x) T_{j-2 l}, \quad B_{j} \neq 0,
$$

with $\omega$-periodic coefficients $B_{l}$, has length $\geq 2 \mathrm{~m}$. 
Proof: Let us assume that $M$ is nonzero and has length $<2 m$. The assumptions on $j$ ensure that $A_{j-2 k}^{j-m-2 k}(x)$ does not vanish identically for $k=0,1,2, \ldots$ Therefore we may subtract from $M$ a suitable linear combination

$$
M_{c}=c_{0} M_{j-m}+c_{1} M_{j-m-2}+\cdots+c_{m-1} M_{j-m-2(m-1)},
$$

so that the result has degree $\leq j-2 m$. We claim that $M=M_{c}$. Since $M_{c}$ has length $\geq 2 m$ unless all $c_{l}$ vanish (see Lemma 4.4), it then follows that $M=0$, contradiction. To prove our claim, let us suppose that $M-M_{c}$ has degree $d \leq j-2 m$. The coefficient of $T_{d}$ in $M-M_{c}$, must have the form $a \phi(x) / \phi(x+d)$, for some constant $a \neq 0$. In particular, it has a pole at $x=m-d$. On the other hand, this coefficient is equal to

$$
-c_{0} A_{d}^{j-m}(x)-\cdots-c_{m-1} A_{d}^{j-3 m+2}(x),
$$

with no contribution from $M$, since $M$ has length $<2 m$. The terms of this sum are of the form const $A_{d}^{l}(x)$ with $d \leq l+m-2$, i.e., $A_{l-m+2 k}^{l}(x)$ with $k \leq m-1$. The pole with largest real part of $A_{l-m+2 k}^{l}$ is $x=-l+m-k$, and this real part is smaller than the real part of $m-d=-l+2 m-2 k$, if $k \leq m-1$. Therefore (3) is regular at $x=m-d$, a contradiction.

Definition: Let $S$ be the involution $S \psi(x)=\psi(-x)$. A difference operator $M$ is symmetric if $M S=S M$. It is antisymmetric if $M S=$ $-S M$.

Lemma 4.6. Suppose that $\gamma$ is irrational. Then any symmetric operator in $D_{\gamma}$ that commutes with $L$ is a polynomial in $L$.

Proof: Suppose that $M \in D_{\gamma}$ is a symmetric difference operator of degree $j$ that commutes with $L$. Let us proceed by induction. If $j=0$, $M$ is a constant multiple of the identity by Lemma 4.3. Let $j>1$. The coefficient of $T_{j}$ has then the form given in Lemma 4.3. If we subtract $c_{j} L^{j}$ from $M$ we obtain a symmetric difference operator of degree at most $j-1$, which is a polynomial in $L$ by the induction hypothesis.

Theorem 4.7. If $\gamma$ is irrational, then all operators in $D_{\gamma}$ commuting with $L$ are polynomials in $L$ and

$$
N=M_{m+1}-S M_{m+1} S .
$$

Proof: We proved this for symmetric operators in Lemma 4.6. Assume that $M \in D_{\gamma}$ is antisymmetric and commutes with $L$. If $M$ has degree $d \geq 2 m+1$, we may subtract a constant multiple of $N L^{d-2 m-1}$, which is antisymmetric and has degree $d$ by Lemma 4.4, to get an operator of 
ALGEBRAIC INTEGRABILITY OF THE TWO-BODY RUIJSENAARS OPERATOIR

smaller degree. This is possible by Lemma 4.3. Thus we may assume that $M$ is antisymmetric and has degree $\leq 2 m$. By subtracting from $M$ a suitable polynomial of $L$ of degree $\leq 2 m$, we get an operator commuting with $L$ and of the form $M^{\prime}=B_{-1}(x) T_{-1}+\cdots+B_{-2 m}(x) T_{-2 m}$. To apply Lemma 4.5, we write $M^{\prime}=M_{o}^{\prime}+M_{e}^{\prime}$, where $M_{o}^{\prime}=B_{-1} T_{-1}+$ $B_{-3} T_{-3}+\cdots$ is the sum of the odd terms. Both $M_{o}^{\prime}, M_{e}^{\prime}$ commute then with $L$, since the property of commutation with $L$ is equivalent to relations involving only even or odd coefficients. But both $M_{o}^{\prime}$ and $M_{e}^{\prime}$ are of negative degree and length $<2 m$, and therefore vanish by Lemma 4.5.

Thus the algebra $A$ is generated by $L$ and $N$. Since $N$ is antisymmetric, its square is symmetric and by Lemma 4.6 we have a relation

$$
N^{2}=Q(L)
$$

for some polynomial $Q$. Comparing the degrees we see that $Q$ has degree $4 m+2$. By Lemma 4.2, $N^{2}$ commutes with $U$, but $L U=-U L$. Since the powers $L^{j}$ of $L$ are linearly independent (they have different degree), it follows that $Q(L)=P\left(L^{2}\right)$ for some polynomial $P$ of degree $2 m+1$.

We are now ready to complete the proof of Theorem 1.2. The fact that $L$ and $N$ commute and obey this relation means that $X \mapsto L$, $Y \mapsto N$ defines a surjective homomorphism of algebras

$$
h: \mathbb{C}[X, Y] /\left(Y^{2}-P\left(X^{2}\right)\right) \mathbb{C}[X, Y] \rightarrow A
$$

Any element of the left algebra is represented uniquely by a polynomial of the form $f(X)+g(X) Y$. Such a polynomial is in the kernel of $h$ if and only if $f(L)+g(L) N$ vanishes. But this means that the symmetric and antisymmetric parts $f(L)$ and $g(L) N$ vanish separately. By considering the coefficient of $T_{j}$ with highest $j$, we see inductively that all coefficients of the polynomial $f$ and $g$ vanish. Thus $\phi$ is an isomorphism.

\section{Eigenvalues}

In this section we compute the eigenvalues of our commuting operators on the Baker-Akhiezer function $\psi(t, x)$. This eigenvalue map maps an element of the algebra of commuting difference operators (i.e., a function on the hyperelliptic curve) to a two-valued function on the Hermite-Bethe curve $Y$ and, as will be shown, realizes the birational equivalence between the hyperelliptic curve and a double covering of the Hermite-Bethe curve.

We start by describing some remarkable properties of the difference operators $M_{l}, l \in \mathbb{C}$. 
Proposition 5.1. For all generic complex l,

$$
L M_{l}=\frac{[l+m]}{[l]} M_{l-1}+\frac{[l-m]}{[l]} M_{l+1}
$$

Proof: If $l$ is generic, then the operator $L M_{l}$ is of degree $l+m+1$ and length $2 m+1$ (Lemma 4.4). Therefore, by Lemma 4.3,

$$
L M_{l}=C_{l} \frac{\phi(x)}{\phi(x+l+m+1)} T_{l+m+1}+\cdots,
$$

up to terms of lower degree. Here $C_{l}$ appears in the coefficient of $T_{l+m}$ in $M_{l}: A_{l+m}^{l}(x)=C_{l} \phi(x) / \phi(x+l+m)$. It follows that by subtracting a suitable multiple of $M_{l+1}$ from $L M_{l}$ we get an operator of degree $\leq l+m-1$. Similarly, $L M_{l}=S L M_{-l} S=C_{-l} \phi(-x) / \phi(-x-l+m+$ 1) $T_{l-m-1}$ plus terms of higher degree, and we may kill the coefficient of $T_{l-m-1}$ by subtracting a multiple of $M_{l-1}$. We conclude that

$$
L M_{l}-\frac{C_{l}}{C_{l+1}} M_{l+1}-\frac{C_{-l}}{C_{-l+1}} M_{l-1}
$$

is an operator of length $<2 m$ commuting with $L$, and thus vanishes by Lemma 4.5. The ratios of $C_{l}$ can easily be computed from the explicit expression for $A_{l+m}^{l}$, and give the desired result.

Thus $M_{l}$, viewed as a function of $l$, is an eigenvector of the $q$-Lamé operator in the space of difference operators with "eigenvalue" $L$.

Lemma 5.2. Let $\omega=1 / \gamma$. Then $M_{l+\omega}=M_{l} T_{\omega}$.

Proof: We have

$$
\begin{aligned}
M_{l+\omega} & =\sum_{k=0}^{m} A_{l+\omega-m+2 k}^{l+\omega} T_{l+\omega-m+2 k} \\
& =\sum_{k=0}^{m} A_{l-m+2 k}^{l} T_{l-m+2 k} T_{\omega}
\end{aligned}
$$

since $A_{l-m+2 k}^{l}$ is $\omega$-periodic as a function of $l$.

\section{Proposition 5.3.}

$$
M_{l} \psi(t, x)=\epsilon_{l}(t) \psi(t, x),
$$

with eigenvalue

$$
\epsilon_{l}(t)=\frac{[2 m] ! \psi(t, l)}{[m] ! \psi(t, m)}
$$


ALGEBRAIC INTEGRABILITY OF THE TWO-BODY RUIJSENAARS OPERATOIR

Proof: Let $\psi(t, x, l)=M_{l} \psi(t, x)$ and denote $\hat{L}$ the $q$-Lamé operator acting on the variable $l$. We then have

$$
\begin{aligned}
\hat{L} \psi(t, x, l) & =\frac{[l+m]}{[l]} M_{l-1} \psi(t, x)+\frac{[l-m]}{[l]} M_{l+1} \psi(t, x) \\
& =L M_{l} \psi(t, x) \\
& =M_{l} L \psi(t, x) \\
& =\epsilon_{L}(t) M_{l} \psi(t, x) .
\end{aligned}
$$

Let $\omega=1 / \gamma$. Let $e^{c}$ be the multiplier of $\psi(t, x): \psi(t, x+\omega)=e^{c} \psi(t, x)$. Then, by Lemma 5.2, we have $\psi(t, x, l+\omega)=e^{c} \psi(t, x, l)$. In other words, both as function of $x$ and as a funcion of $l, \psi(t, x, l)$ is an eigenfunction of $L$ with the same eigenvalue and multiplier. If $t$ is generic, there is only one such eigenfunction up to normalization. Thus

$$
\psi(t, x, l)=f(t) \psi(t, l) \psi(t, x)
$$

for some $f(t)$. On the other hand, we know that if $l=m, M_{m}=$ $[2 m] ! /[m]$ ! times the identity. This determines $f$ and we get

$$
\psi(t, x, l)=\frac{[2 m] !}{[m] !} \frac{\psi(t, l) \psi(t, x)}{\psi(t, m)}
$$

As a corollary, we see that the relation of Theorem 1.3 is a special case of more general product rules:

Corollary 5.4. For generic $l, m \in \mathbb{C}$,

$$
M_{l} M_{k}=\sum_{j} A_{j}^{l}(k) M_{k+j},
$$

where $A_{j}^{l}, j=l-m, l-m+2, \ldots, l+m$, are the coefficients defined in Section 因.

Proof: By Lemma 3.4, it is sufficient to prove this identity for the eigenvalues $\epsilon_{l}(t)$. We first note that Proposition 5.3 can be rewritten, after replacing $x$ by $k$, as

$$
\sum_{j} A_{j}^{l}(k) \psi(t, k+j)=\epsilon_{l}(t) \psi(t, k) .
$$


Hence,

$$
\begin{aligned}
\sum_{j} A_{j}^{l}(k) \epsilon_{k+j}(t) & =\frac{[2 m] !}{[m] !} \sum_{j} A_{j}^{l}(k) \frac{\psi(t, k+j)}{\psi(t, m)} \\
& =\frac{[2 m] !}{[m] !} \epsilon_{l}(t) \frac{\psi(t, k)}{\psi(t, m)} \\
& =\epsilon_{l}(t) \epsilon_{k}(t) .
\end{aligned}
$$

Corollary 5.5. Let $N=M_{m+1}-M_{-m-1}$. Then

$$
N \psi(t, x)=\epsilon_{N}(t) \psi(t, x) \text {, }
$$

where

$\epsilon_{N}(t)=\frac{[2 m] !}{[m] !}\left(e^{\gamma c} \prod_{j=1}^{m} \frac{\left[m+t_{j}+1\right]}{\left[m+t_{j}\right]}-e^{-\gamma c} \prod_{j=1}^{m} \frac{\left[m-t_{j}+1\right]}{\left[m-t_{j}\right]}\right), \quad e^{\gamma c}=\sqrt{b_{j}(t)}$.

The map $t \mapsto\left(\epsilon_{L}(t), \epsilon_{N}(t)\right)$ defines a birational isomorphism from the double covering $Y_{2}$ of the Hermite-Bethe curve to the curve $\{(X, Y) \in$ $\left.\mathbb{C}^{2} \mid Y^{2}=P\left(X^{2}\right)\right\}$.

Proof: The expression for the eigenvalue is taken from Proposition 5.3.

By construction, the function $\epsilon_{L}(t)$ is a two-to-one rational function from the closure of $Y_{2}$ onto $\mathbb{P}^{1}$. The two points in $\epsilon_{L}^{-1}(p)$ for generic $p \in \mathbb{P}^{1}$ are related by the symmetry $(t, c) \mapsto(-t,-c)$. The eigenvalue $\epsilon_{N}$ is odd under this symmetry, and we thus have a one-to-one (at generic points) map from $Y_{2}$ to the hyperelliptic curve.

Remarks.

1. The hyperelliptic curve $Y^{2}=P\left(X^{2}\right)$ has a double point at infinity which is resolved into the two points $P_{+}$and $P_{-}$of the double covering $Y_{2}$ of the Bethe-Hermite curve. These are the points at which the eigenvalues of the commuting operators have poles. Our results can be considered as a degenerate case of the difference version of Krichever's construction: to a smooth projective curve $C$ of genus $g$, two points $P_{+}, P_{-}$on it and a generic effective divisor $D$ of degree $g$, Krichever [K] associates a Baker-Akhiezer function $\psi(p, x)$, a properly normalized meromorphic function of $p \in C$ and $x \in \mathbb{Z}$ with divisor (the formal sum-with-multiplicities of the poles minus the zeros) $x\left(P_{+}-P_{-}\right)+D$. To each meromorphic function $f$ on $C$ which is regular on $C-\left\{P_{+}, P_{-}\right\}$is associated a difference operator $M_{f}$ with integer steps, for which $\psi(p, \cdot)$ is an eigenfunction with eigenvalue $f(p)$, and $f \mapsto M_{f}$ is an algebra homomorphism. In particular, if $P$ is a polynomial of odd degree 
ALGEBRAIC INTEGRABILITY OF THE TWO-BODY RUIJSENAARS OPERATOIR

$2 m+1$ without multiple roots, then the curve $Y^{2}=P\left(X^{2}\right)$ can be compactified to a smooth hyperelliptic curve by adding two points $P_{+}, P_{-}$at infinity, and one has, upon choosing a divisor, a pair of commuting difference operators $M_{X}$ and $M_{Y}$. Note that in general the Baker-Akhiezer function is written in terms of the Riemann theta function of the hyperelliptic curve, whereas in this case it can be written purely in terms of an elliptic curve.

2. Note the analogy with "fusion algebras". Let $N_{j k}^{i}=\operatorname{dim} \operatorname{Hom}\left(V_{i}, V_{j} \otimes\right.$ $V_{k}$ ) be the dimensions of the space of homomorphisms of, say, representations of a simple Lie group $G$. Here $V_{i}$ are irreducible finite dimensional representations, labeled by their highest weight $i \in P^{+}$. Let us introduce difference operators $M_{l}$ acting on functions on $P^{+}$by the formula $M_{l} f(i)=\sum_{j} N_{l j}^{i} f(j)$. The commutativity and associativity of the tensor product imply that the operators $M_{l}$ commute with each other. Moreover, we have $M_{l} M_{k}=\sum_{j} N_{l k}^{j} M_{j}$, cf. Corollary 5.4. Let $\psi(t, j)=\operatorname{tr}_{V_{j}}(t)$ be the character of the representation $V_{j}$. It is a function of $t \in T / W$, the quotient of a Cartan torus by the Weyl group. Then, for fixed $t$, the function $\psi(t, j)$ of $j$ is a common eigenfunction of all difference operators $M_{l}$ :

$$
M_{l} \psi(t, \cdot)=\epsilon_{l}(t) \psi(t, \cdot), \quad \epsilon_{l}(t)=\frac{\psi(t, l)}{\psi(t, 0)},
$$

cf. Proposition 5.3. If we replace $G$ by a quantum group at root of unity, the same formulae apply, except that $P^{+}$is replaced by a finite subset and $T$ is replaced by the set of points of a finite order $N$, depending on the order of the root of unity, in the Cartan torus. In this case, $\psi(t, j)$ has a remarkable interpretation in terms of representations of $S L(2, \mathbb{Z})$, discovered by E. Verlinde [V], in the context of conformal field theory. Is there a similar interpretation in our case?

\section{The Galois Group}

We compute the "difference Galois group" of the q-Lamé equation. This group is a difference analogue of the differential Galois group of differential equations. The computation is motivated by the recent result of Braverman, Etingof and Gaitsgory BEG, who, in the differential case, characterized algebraic integrability by the Abelian property of the Galois group.

Definition: A difference field is a field $F$ together with an automorphism $T \in \operatorname{Aut}(F)$. An extension $E \subset F$ of difference fields is a subfield $E$ of a difference field $F$ such that $T(E) \subset E$. An automorphism of a 
difference field $F$ is an automorphism of the field $F$ commuting with $T$. The Galois group of an extension $E \subset F$ of difference fields is the group of those automorphisms of $F$ which restrict to the identity on E.

Let us now consider our difference equation $L \psi=\epsilon \psi$ for fixed generic $\epsilon$. If we make the change of variables $\psi(x)=u(x) \prod_{j=1}^{m}[x-j]$, the equation becomes

$$
u(x+1)+\frac{[x+m][x-m-1]}{[x][x-1]} u(x-1)=\epsilon u(x) .
$$

This difference equation has coefficients in the field $E$ of elliptic functions with periods $\omega, \omega^{\prime}$. The field $E$ is a difference field with $T$ the shift $T f(x)=f(x+1)$.

Let $F$ be the differential field generated over $E$ by the meromorphic solutions of the difference equation (A). It is the field of all rational functions in the solutions and their images by $T^{j}, j \in \mathbb{Z}$, with coefficients in $E$.

It follows from Theorem 3.1 that the solutions of (4) are linear combinations of $u_{+}(x)$ and $u_{-}(x)=u_{+}(-x)$ with coefficients in $K$, the field of meromorphic 1-periodic functions. The solution $u_{+}$has the form

$$
u_{+}(x)=e^{c \gamma x} \prod_{j=1}^{m} \frac{\left[x+t_{j}\right]}{[x-j]} .
$$

Theorem 6.1. Let $\gamma \in \mathbb{R}-\mathbb{Q}$ and $\epsilon$ be generic. Then the Galois group of the extension $E \subset F$ of difference fields is isomorphic to the group $K^{\times}$of non-zero meromorphic 1-periodic functions on the complex plane. A function $h \in K^{\times}$corresponds to the automorphism acting on solutions by $u_{ \pm}(x) \mapsto h(x)^{ \pm 1} u_{ \pm}(x)$.

To prove the theorem, we first need some auxiliary results.

Lemma 6.2. If $\Phi$ is an element of the Galois group then $\Phi\left(u_{ \pm}\right)=$ $h^{ \pm 1} u_{ \pm}$, for some $h \in K^{\times}$.

Proof: The function $u_{+}(x)$ has constant multipliers as $x$ is shifted by $\omega$ or $\omega^{\prime}$. Thus $T u_{+} / u_{+}$is an elliptic function. It follows that if a Galois automorphism sends $u_{+}$to a function $\tilde{u}_{+}$, then $T \tilde{u}_{+} / \tilde{u}_{+}=T u_{+} / u_{+}$. Thus $\tilde{u}_{+}=h u_{+}$for some function $h \in K^{\times}$.

Similarly, $u_{+}(x) u_{-}(x)$ is elliptic. Thus if $u_{+}$is sent to $h u_{+}$, then $u_{-}$ is sent to $h^{-1} u_{-}$.

Let $\hat{E}$ be the field generated by $K$ and $E$. 
ALGEBRAIC INTEGRABILITY OF THE TWO-BODY RUIJSENAARS OPERATOIR

Lemma 6.3. $F=\hat{E}\left(u_{+}\right)$, i.e., the field $F$ consists of rational functions in $u_{+}$with coefficients in $\hat{E}$.

Proof: Notice first that $K \subset F$ : an element $h \in K$ is the ratio of solutions $h u_{+} / u_{+}$. It remains to show that every element of $F$ can be written as a rational function in $u_{+}$with coefficients in $\hat{E}$. But the proof of the preceding lemma shows that $u_{-}$and $T u_{+}$and thus all solutions, as well as all their images by $T^{j}, j \in \mathbb{Z}$, are rational functions in $u_{+}$with coefficients in $\hat{E}$.

Lemma 6.4. $u_{+}$is transcendental over $\hat{E}$.

Proof: This means that $u_{+}$is not the solution of any non-trivial polynomial equation with coefficients in $\hat{E}$. Suppose that there is such an equation

$$
P\left(x, u_{+}(x)\right)=\sum A_{j}(x) u_{+}(x)^{j}=0, \quad A_{j} \in \hat{E} .
$$

Since $\omega$ is real, there exists a stricly increasing sequence of integers $n_{1}, n_{2}, \ldots$, so that the distance between $n_{l} \omega$ and the lattice of integers converges to zero. Let $x$ be any generic complex number and set $x_{l}=x+n_{l} \omega$. Then $\lim _{l \rightarrow \infty} A_{j}\left(x_{l}\right)=A_{j}(x)$, for all $j$. On the other hand, $u_{+}\left(x_{l}\right)=C^{n_{l}} u_{+}(x)$, for some non-trivial constant $C$. Since $P\left(x_{l}, u_{+}\left(x_{l}\right)\right)=0$ for all $l$, it follows that all coefficients $A_{j}(x)$ vanish at $x$. But $x$ is arbitrary. Therefore all coefficients vanish identically, a contradiction.

The proof of Theorem 6.1 can now be completed. What is left to prove is that, for every $h \in K^{\times}$, there exists a unique Galois automorphism sending $u_{+}$to $h u_{+}$.

The uniqueness follows from Lemma 6.2 and Lemma 6.3.

To prove existence, we have to show that for all rational functions $f \in \hat{E}(X)$ of one indeterminate, the map

$$
f\left(u_{+}\right) \mapsto f\left(h u_{+}\right),
$$

is well-defined, i.e., independent of the choice of the function $f$ used to represent an element $f\left(u_{+}\right) \in F$, and that it defines an automorphism of difference fields.

The map is well-defined: if $f\left(u_{+}\right)=g\left(u_{+}\right)$for rational functions $f=p / q, g=r / s$, then $u_{+}$is a solution of the polynomial equation $p s-q r=0$. By Lemma 6.4, this equation must be trivial, meaning that $f=g$ in $\hat{E}(X)$. In particular $f\left(h u_{+}\right)=g\left(h u_{+}\right)$.

It is clear that (5) defines an automorphism of fields with inverse $f\left(u_{+}\right) \mapsto f\left(u_{+} / h\right)$. Let us show that it is an automorphism of difference 
fields. We have $T f\left(u_{+}\right)=\bar{f}\left(u_{+}\right)$. The rational function $\bar{f}(X)$ has the form $(T f)(a X)$, where $a=T u_{+} / u_{+} \in E$ and $T f$ is obtained from $f$ by acting with $T$ on the coefficients. We have to show that $T\left(f\left(h u_{+}\right)\right)=$ $\bar{f}\left(h u_{+}\right)$. But since $T\left(h u_{+}\right)=h T\left(u_{+}\right)$, we have

$$
T\left(f\left(h u_{+}\right)\right)=(T f)\left(h T\left(u_{+}\right)\right)=(T f)\left(h a u_{+}\right)=\bar{f}\left(h u_{+}\right) .
$$

The proof is complete.

Remark. Our construction is a special case of a more general construction in higher dimension: a difference field in $n$ dimensions is a field $F$ together with $n$ commuting automorphisms $T_{1}, \ldots, T_{n}$. Extensions and Galois groups are defined as obvious generalizations of the $n=1$ case. Suppose that $F$ is a field of functions $f\left(x_{1}, \ldots, x_{n}\right)$ and $T_{i}$ are shift operators

$$
T_{i} f\left(x_{1}, \ldots, x_{n}\right)=f\left(x_{1}, \ldots, x_{i}+a_{i}, \ldots, x_{n}\right), \quad i=1, \ldots, n .
$$

Let us say that the function $g$ is elementary with respect to the difference field $F$ if there are functions $f_{1}, \ldots, f_{n}$ in $F$ such that $g$ satisfies the equations

$$
T_{i} g / g=f_{i}, \quad i=1, \ldots, n
$$

Let

$$
L_{j} F\left(x_{1}, \ldots, x_{n}\right)=0, \quad j=1, \ldots, k,
$$

be a system of linear difference equations with coefficients in the difference field $F$. Assume that the space of solutions has a basis consisting of elementary functions. Then the Galois group of this system is Abelian.

\section{REFERENCES}

[BEG] A. Braverman, P. Etingof and D. Gaitsgory, Differential Galois groups and algebraic integrability of quantum integrable systems, alg-geom/9607012

[CV] O. Chalykh and A. Veselov, Commutative rings of differential operators and Lie algebras, Commun. Math. Phys. 126 (1990), 597-611

[DMN] B. A. Dubrovin, V. B. Matveev and S. P. Novikov, Nonlinear equations of Korteweg-de Vries type, finite-zone potentials and abelian varieties, Russ. Math. Surv. 31 (1976), 51-125

[FV] G. Felder and A. Varchenko, Algebraic Bethe ansatz for the elliptic quantum group $E_{\tau, \eta}\left(s l_{2}\right)$, to appear in Nucl. Phys. B

[K] I. Krichever, Algebraic curves and difference equations, Russ. Math. Surv. 33:4 (1978), 255-356

[KZ] I. Krichever and A. Zabrodin, Spin generalization of the RuijsenaarsSchneider model non-abelian 2D Toda Chain and representations of Sklyanin algebra, hep-th/9505039

[R] S. N. M. Ruijsenaars, Complete integrability of relativistic CalogeroMoser systems and elliptic function identities, Commun. Math. Phys. 110 (1987), 191-213 


\section{ALGEBRAIC INTEGRABILITY OF THE TWO-BODY RUIJSENAARS OPERATOIR}

[S] E. Sklyanin, On some algebraic structures related to the Yang-Baxter equation. Representations of the quantum algebra, Fun. Anal. Pril. 17:4 (1983) 34-48

[V] E. Verlinde, Fusion rules and modular transformations in $2 D$ conformal field theory, Nucl. Phys. B300[FS22] (1987), 360-376

[WW] E. T. Whittaker and G. N. Watson, A course of modern analysis, Cambridge University Press, 1915 